\title{
Author Correction: Drivers of seedling establishment success in dryland restoration efforts
}

Nancy Shackelford (D), Gustavo B. Paterno (D), Daniel E. Winkler, Todd E. Erickson, Elizabeth A. Leger, Lauren N. Svejcar, Martin F. Breed (D), Akasha M. Faist, Peter A. Harrison (D), Michael F. Curran, Qinfeng Guo (D), Anita Kirmer (D), Darin J. Law, Kevin Z. Mganga, Seth M. Munson (D), Lauren M. Porensky, R. Emiliano Quiroga, Péter Török (D), Claire E. Wainwright, Ali Abdullahi, Matt A. Bahm, Elizabeth A. Ballenger, Nichole Barger, Owen W. Baughman, Carina Becker (D), Manuel Esteban Lucas-Borja, Chad S. Boyd, Carla M. Burton, Philip J. Burton (D, Eman Calleja, Peter J. Carrick, Alex Caruana (D), Charlie D. Clements, Kirk W. Davies, Balázs Deák Di, Jessica Drake, Sandra Dullau (iD, Joshua Eldridge, Erin Espeland, Hannah L. Farrell, Stephen E. Fick, Magda Garbowski, Enrique G. de la Riva, Peter J. Golos D, Penelope A. Grey, Barry Heydenrych, Patricia M. Holmes D, Jeremy J. James, Jayne Jonas-Bratten (D, Réka Kiss, Andrea T. Kramer, Julie E. Larson, Juan Lorite D, C. Ellery Mayence, Luis Merino-Martín (D), Tamás Miglécz, Suanne Jane Milton (D), Thomas A. Monaco, Arlee M. Montalvo, Jose A. Navarro-Cano, Mark W. Paschke, Pablo Luis Peri, Monica L. Pokorny, Matthew J. Rinella, Nelmarie Saayman, Merilynn C. Schantz, Tina Parkhurst, Eric W. Seabloom (D), Katharine L. Stuble, Shauna M. Uselman, Orsolya Valkó (D, Kari Veblen D, Scott Wilson, Megan Wong, Zhiwei Xu D and Katharine L. Suding (iD

Correction to: Nature Ecology \& Evolution https://doi.org/10.1038/s41559-021-01510-3, published online 22 July 2021.

In the version of this Article originally published, the surname of author Tina Parkhurst was incorrectly written as Schroeder. This has now been corrected.

Published online: 28 July 2021

https://doi.org/10.1038/s41559-021-01544-7

(C) The Author(s), under exclusive licence to Springer Nature Limited 2021 